\title{
Open Total Talar Dislocation with Extrusion: Tips and Tricks in Management
}

\author{
Jean P Filler Leung ${ }^{1}$, Bernardino Alpuerto $\|^{2}$, Ilian Eusebio ${ }^{3}$
}

\begin{abstract}
Open total talar dislocations are rare but potentially catastrophic injuries of the hindfoot. Successful management involves following the principles of open fracture management-thorough debridement, reduction, stabilization, and wound management. We detail our surgical technique in managing such a case, with specific tips on each step that may help others in the management of such cases.

Keywords: Early surgery, External fixation, Talar extrusion.

Journal of Foot and Ankle Surgery (Asia Pacific) (2021): 10.5005/jp-journals-10040-1175
\end{abstract}

\section{CASE}

A 33-year-old man fell down a 30-m ravine while harvesting vegetables, sustaining an open right foot injury. Due to the distance from the main roads, he was brought to the emergency room 20 hours after his injury. Vital signs were normal and stable. A 12-cm gaping wound over the medial aspect of his $\mathrm{R}$ hindfoot with a partially extruded bone and fairly severe wound contamination. The distal foot was slightly cool with a moderate capillary refill. Our clinical diagnosis is an open total talar dislocation with medial extrusion of the talar head of his right ankle, which was confirmed with a CT scan (Fig. 1).

\section{Management}

Initial documentation of injury with clinical pictures, X-rays, and CT scan was done at the ER. The patient was given antitetanus prophylaxis and started on cefazolin $1 \mathrm{~g} \mathrm{IV} \mathrm{q} 8 \mathrm{~h}$, gentamicin $80 \mathrm{mg}$ IV q 8h, and metronidazole $500 \mathrm{mg}$ IV q $8 \mathrm{~h}$ to cover for anaerobes. Gross decontamination of the wound was done with a wash of $2 \mathrm{~L}$ plain NSS, and the wound was covered with a moist dressing while awaiting OR.

The patient underwent a formal debridement 24 hours after the injury. After copious irrigation with $10 \mathrm{~L}$ of plain NSS into the joint, removal of torn soft tissues partially attached to the extruded talus, and loose osteocartilaginous fragments was performed. Intraoperative cultures were obtained. The extruded talus was carefully assessed and judged to be sufficiently clean to permit replantation. Replantation of the talus was done via the following sequence (Fig. 2):

- Application of a Shantz pin or a transcalcaneal Steinmann pin across the body of the calcaneus to allow longitudinal traction of the calcaneus.

- Application of a partially threaded $4 \mathrm{~mm}$ Shantz screw into the medial portion of the talar head to serve as a joystick and facilitate reduction after distraction and valgus opening of hindfoot with transcalcaneal pins.

- Soft tissue interposition at the anterior part of the talus space was retracted to prevent obstruction-in our case, a Homan retractor was used to retract the tibialis anterior tendon and portions of the anterior deltoid ligament anterosuperior to
${ }^{1}$ Department of Orthopedics, Notre Dame de Chartres Hospital, Baguio, Philippines

2,3 Department of Orthopedics, University of the Philippines, Metro Manila, Philippines

Corresponding Author: Jean P Filler Leung, Department of Orthopedics, Notre Dame de Chartres Hospital, Baguio, Philippines, Phone: +63 9209053170, e-mail: jpleung@hotmail.com

How to cite this article: Filler Leung JP, Alpuerto II B, Eusebio I. Open Total Talar Dislocation with Extrusion: Tips and Tricks in Management. J Foot Ankle Surg (Asia Pacific) 2021;8(4):206-208.

Source of support: Nil

Conflict of interest: None

accommodate the extruded talar head. In some cases, the talar head could be button-holed in-between the posterior tibialis and flexor digitorum longus tendons blocking reduction of the talus back into the mortise and these tendons should be retracted as well to be able to reduce the talus back into the mortise.

- The extruded talus was rotated and reduced back into its place into the joint. It is important to do this as atraumatic as possible to avoid inadvertent injury to the neurovascular bundles posteriorly and also prevent further damage to the articular surfaces.

- Radiographic confirmation of the reduction was done.

After reduction was confirmed, temporary stabilization of the reduced talus was done with $2 \mathrm{~mm} k$-wires. A biplanar delta fixator with centrally threaded Steinman pins applied at the level of the distal tibia, calcaneal body, and navicular was applied (Fig. 3). This fixation provided adequate stabilization of the reduced dislocation as well as providing adequate access for wound management. The wound was left open and initially packed with a wet to dry dressing.

The patient underwent a second look debridement and wash out after 48 hours with a note of some sero-purulent discharge. Six liters of NSS was used and a locally developed vacuum-assisted dressing was applied to help draw out fluids and maintained for 5 days (Fig. 4). Bacterial cultures from the initial OR grew Staphylococcus sp. and the patient was shifted to levofloxacin $750 \mathrm{mg}$ OD for 6 weeks.

(o) The Author(s). 2021 Open Access This article is distributed under the terms of the Creative Commons Attribution 4.0International License (https://creativecommons. org/licenses/by-nc/4.0/), which permits unrestricted use, distribution, and non-commercial reproduction in any medium, provided you give appropriate credit to the original author(s) and the source, provide a link to the Creative Commons license, and indicate if changes were made. The Creative Commons Public Domain Dedication waiver (http://creativecommons.org/publicdomain/zero/1.0/) applies to the data made available in this article, unless otherwise stated. 

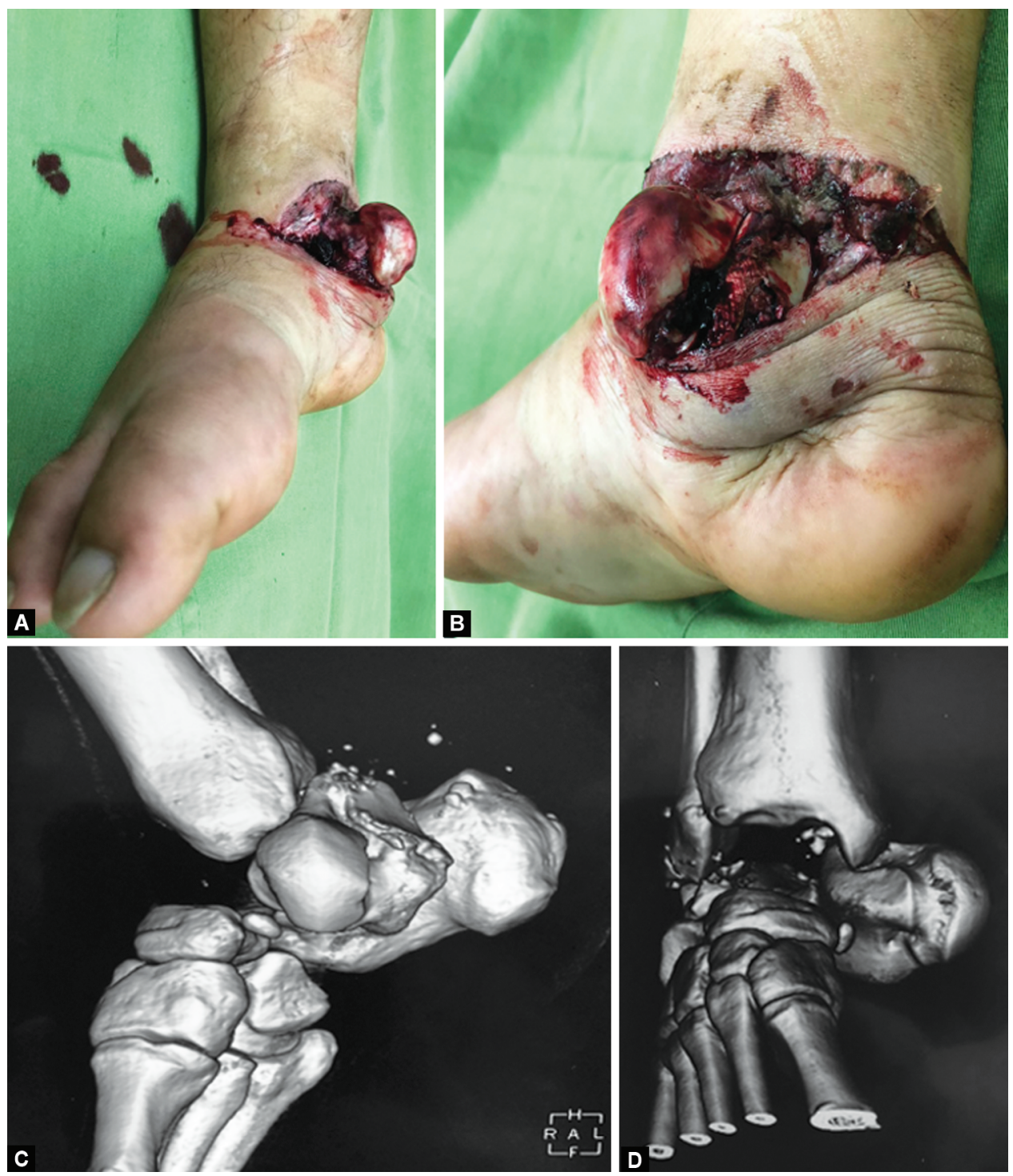

Figs $1 A$ to D: (A and B) Clinical images of the patient's right foot; (C and D) CT scan showing medial total talar dislocation with loose bodies in the joint

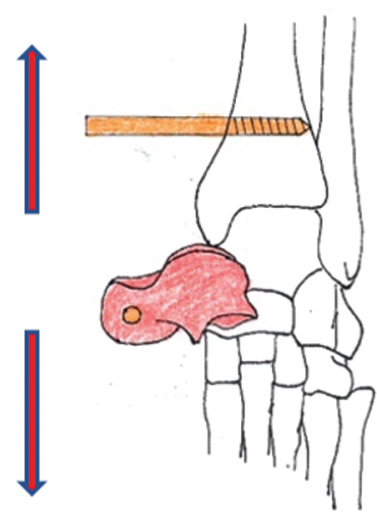

A

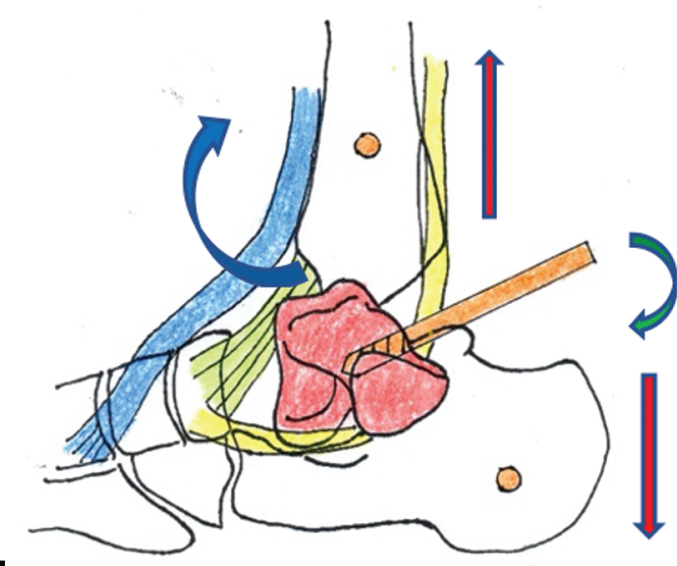

B

Figs $2 \mathrm{~A}$ and B: Illustration of medial open dislocation of the talus including directions of reduction. (A) AP view of ankle showing dislocation of talus in a posteromedial direction. Schantz pin (orange pin) could be placed at distal tibia for axial distraction as showed by red arrows. Schantz pin (orange circle) was placed in the talar head to aid in the reduction of the talus back into the joint; (B) Medial view of ankle. Aside from axial distraction (red arrows), derotation of the talus using the Schantz pin in the talar head should be done as shown by the green arrow, and retraction of the anterior tibialis (blue structure) and anterior deltoid ligaments (light green structure) anterosuperiorly (blue arrow) should also be done to reduce the talus back in the mortise. The yellow structure is a posterior tibialis tendon 


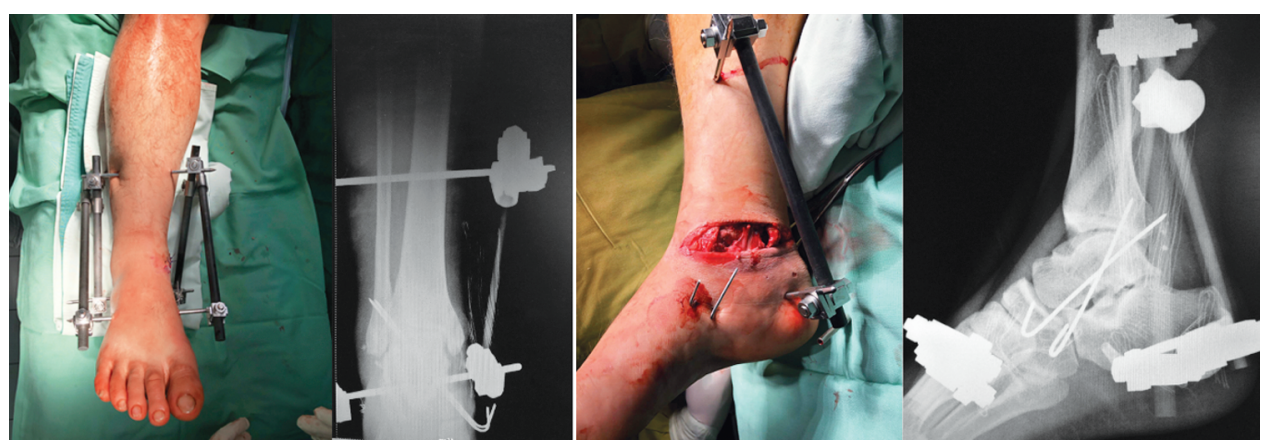

Fig. 3: Clinical and radiographic images after the initial debridement and fixation

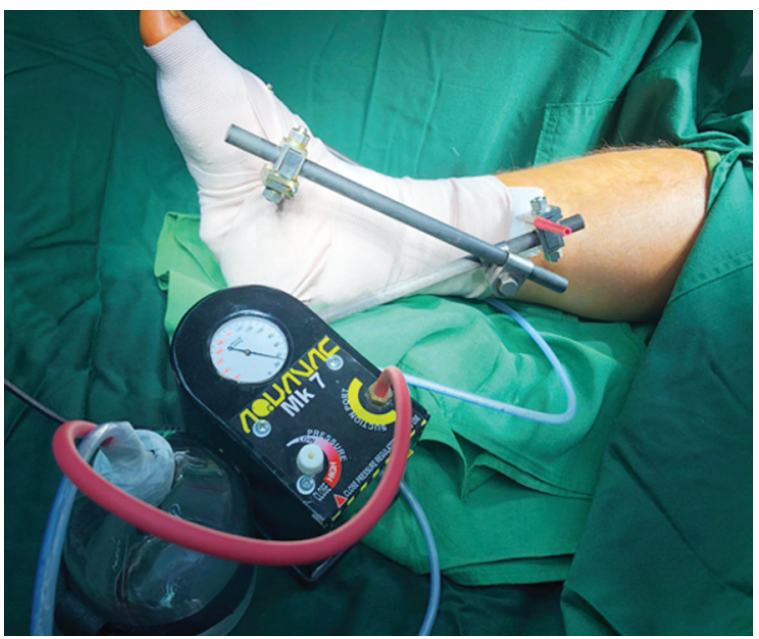

Fig. 4: Clinical photograph after the second debridement showing the locally developed vacuum device

The patient was discharged improved and the wound was dressed with occlusive dressings weekly at an outpatient clinic. The fixator was removed after 10 weeks and physical therapy with a range of motion exercises started. Weight-bearing will be delayed until 12 weeks postoperatively.

\section{Discussion}

Total talar dislocations are rare injuries, comprising roughly $0.6 \%$ of all dislocations and $2 \%$ of all talar injuries. They may be open or closed and may or may not be associated with fractures.

Management of open total talar dislocations includes broadspectrum antibiotics, antitetanus prophylaxis, and early aggressive debridement of the wound and extruded fragment. During debridement, all foreign body contaminants, loose chondral bodies, and ligament stumps that may hamper reduction should be identified and removed. The decision to reduce or excise the talus is influenced by the severity of wound contamination, duration from the time of injury, the severity of concomitant fractures, and the patient's overall status. Historically, cases reported involved removal of the extruded talus and early tibiocalcaneal fusion, however, functional results were poor. More recent studies have focused on the reduction and stabilization of the extruded talus, associated joints, and concomitant fractures. ${ }^{1-6}$ A spanning external fixator may be added to the construct. Severe cartilage damage necessitates fusion of the involved joints. Long-term complications include infection (10\%), posttraumatic arthritis (14-30\%), and avascular necrosis (24-26\%). ${ }^{7}$ The presence of these complications may necessitate secondary procedures and may adversely affect long-term functional outcome.

\section{References}

1. AIMaeen BN, EIMaghrby IS, Alnour MK, etal.Complete revascularization of reimplanted talus after isolated total talar extrusion: a case report. Cureus 2020;12(5):e7947. DOI: 10.7759/cureus.7947.

2. Cocjin HGB, Jingco JKP, Tumaneng FDC, et al. Wound-healing following a negative-pressure wound therapy with use of a locally developed AquaVac system as compared with the vacuum-assisted closure (VAC) system. J Bone Joint Surg Am 2019;101(22):1990-1998. DOI: 10.2106/JBJS.19.00125.

3. Kwak JM, Heo SK, Jung GH. Six-year survival of reimplanted talus after isolated total talar extrusion: a case report. J Med Case Rep 2017;11(1):348. DOI: 10.1186/s13256-017-1517-7.

4. Mohammad HR, A'Court J, Pillai A. Extruded talus treated with reimplantation and primary tibiotalocalcaneal arthrodesis. Ann $R$ Coll Surg Engl 2017;99(4):e125-e127. DOI: 10.1308/rcsann.2017.0042.

5. Pavić R. Talocalcaneal transfixation in total dislocation of the talus and subtalar dislocations. Mil Med 2009;174(3):324. DOI: 10.7205/ milmed-d-00-8408.

6. Vaienti L, Maggi F, Gazzola R, et al. Therapeutic management of complicated talar extrusion: literature and case report. J Orthopaed Traumatol 2011;12(1):61-64. DOI: 10.1007/s10195-011-0129-z.

7. Weston JT, Liu X, Wandtke ME, et al. Review article: a systematic review of total sislocation of the talus. Orthopaedic Surgery 2015;7(2):97-101. DOI: $10.1111 /$ os.12167. 